\title{
IRWIN NUMBER AND DUCTILE-BRITTLE FRACTURE TRANSITION
}

\section{Ya-Pu Zhao}

Lab for Non-Linear Mechanics of Continuous Media, Institute of Mechanics Chinese Academy of Sciences, Beijing 100080, People's Republic of China Tel: (010) 2561834

A change in fracture mode, or from fracture to no fracture, is generally termed a fracture transition [1]. As an important nonlinear phenomenon of material failure, the existence of ductile-brittle fracture transition is due to several factors; it may be induced by specimen size, change of environmental temperature, loading rate, etc. It is obvious that the environmental conditions associated with a ductile-brittle transition in a material are an important factor in design, and it is important to ensure that a ductile-brittle fracture transition is not encountered when scaling up the results from small-scale models to predict the behavior of full-scale prototypes. The importance of this problem has been recently emphasized many times by review articles and monographs (see for example $[2,3]$ ).

For convenience, the scaling laws concerning both brittle and ductile fracture mechanics are given. For brittle fracture, extension of a crack requires

$$
f(\sigma, 1, E, \gamma, \nu)=0,
$$

where $\sigma$ is the stress, $\imath$ the characteristic length of the cracked structure, $E$ the Young's modulus, $\gamma$ the surface energy, and $v$ the Poisson's ratio. According to Griffith, the extension of a crack is the transformation of elastic energy to surface energy, then

$$
f\left(\frac{\sigma^{2}}{E}, l, \gamma, v\right)=0 .
$$

By dimensional analysis we have

$$
\frac{\sigma^{2} l}{E \gamma}=f(v) .
$$

From (3) we know that Poisson's ratio is the only similarity parameter for brittle fracture. If both geometric and material similarities are satisfied, the scaling law concerning fracture stress between prototype and model is 


$$
\left(\frac{\sigma^{(p)}}{\sigma^{(m)}}\right)^{2}=\frac{(E \gamma)^{(p)}}{(E \gamma)^{(m)}}\left(\frac{\mathrm{i}^{(p)}}{\mathrm{l}^{(m)}}\right)^{-1},
$$

where $(m)$ and $(p)$ represent model and prototype, respectively. Alternatively, (4) may be written in the following form

$$
\frac{\sigma^{(p)}}{\sigma^{(m)}}=\frac{K_{c}^{(p)}}{K_{c}^{(m)}}\left(\frac{\mathfrak{l}^{(p)}}{\mathfrak{l}^{(m)}}\right)^{-1 / 2},
$$

where $\mathrm{K}_{\mathrm{c}}$ denotes fracture toughness of the material.

For ductile fracture, the extension of a crack requires

$$
f\left(\sigma, K_{c}, \sigma_{y}, l, v\right)=0
$$

where $\sigma_{y}$ is the yield stress of the material. By $\Pi$-theorem, there is an additional similarity parameter for ductile fracture

$$
\mathrm{I}=\frac{\sigma_{y} \mathrm{l}^{1 / 2}}{K_{c}}
$$

This similarity parameter was termed the Irwin number by Barenblatt [4] to recognize this fundamental contribution to fracture mechanics. The Irwin number is physically the square root of the ratio of the characteristic length of the structure to the magnitude of the length of the plastic zone near the crack tip, which is an intrinsic structural parameter of the material. The Irwin number is a basic similarity parameter in ductile fracture. In fact, it is possible to define small-scale yielding (SSY) and large-scale yielding (LSY) with the aid of the Irwin number. If similar conditions $V^{(m)=} V^{(p)}$ and $I^{(m)}=I^{(p)}$ are satisfied, the scaling law conerning fracture stress between prototype and model is also given by (5). In this case, (6) can be written in the following form

$$
f\left(\frac{\sigma}{\sigma_{y}}, \mathrm{I}, v\right)=0 .
$$

Generally speaking, brittle fracture is stress intensity factor K-dominant, and ductile fracture is flow stress controlled. The famous Ludwik-DavidenkovOrowan hypothesis (illustrated in Fig. 1) assumed that brittle fracture and plastic flow are two independent processes, the intersection of the two curves defines the ductile-brittle fracture transition. In this case, letting $\sigma=\sigma_{y}$ in (8), we have the transition condition

$$
f(1, \mathrm{I}, \mathrm{v})=0 .
$$


For a specific material at a given working condition (environmental temperature, loading rate, configuration, etc.), the ductile-brittle fracture transition condition can be expressed as

$$
\mathrm{I}=\mathbb{I}_{c r} \text {. }
$$

Thus, ductile-brittle fracture transition can be considered when the basic similarity parameter - the Irwin number reaches a critical value, or the ratio of the chracteristic length of the cracked structure to the size of the plastic zone near the crack tip reaches a critical value. With a given material and working condition, $\mathrm{I}_{c r}$ depends on the configuration of the specimen. It is obvious that brittle fracture occurs when

$$
\mathrm{I}>\mathrm{I}_{c r} \text {, }
$$

and ductile fracture occurs when

$$
\mathrm{I}<\mathrm{I}_{c r} \text {. }
$$

The advantage of using the Irwin number to model ductile-brittle fracture transition is two-fold: first, the Irwin number is a similarity parameter in ductile fracture between models and prototypes, ductile-brittle fracture transition will occur simultaneously for both models and prototypes when their Iwin numbers are identical. Second, the Irwin number is a non-dimensional one, different structures can be easily compared for ductile-brittle fracture transitions by using this dimensionless number.

For the famous Kendall's problem [2,3,5,6,7] illustrated in Figs. 2 and 3, the critical value of the Irwin number is

$$
I_{c r}=\frac{4}{\sqrt{3}} \text {. }
$$

For the double cantilever specimen [8], shown in Fig. 4, we have

$$
\mathrm{I}_{c r}=\sqrt{3} \text {. }
$$

Acknowledgement: The support from the National Natural Science Foundation of China and the Foundation of the CAS president are gratefully acknowledged.

\section{REFERENCES}

[1] K.E. Puttick, Journal of Physics D, Applied Physics 13 (1980) 12249-2262.

[2] N. Jones, Applied Mechanics Review 42 (1989) 95-115.

[3] N. Jones, Structural Impact, Cambridge University Press (1989). 
[4] G.I. Barenblatt, in Modeling of Defects ana Fracture Mechanics, Springer-Verlag (1993).

[5] K. Kendall, Journal of Material Science 11 (1976) 1267-1269.

[6] K. Kendall, Proceedings of the Royal Society (London) A361 (1978) 245-263.

[7] K. Kendall, Nature 272 (1978) 710-711.

[8] I.M. Ward, Mechanical Properties of Solid Polymers, Wiley-Interscience, 2nd edn. (1983).

23 November 1995

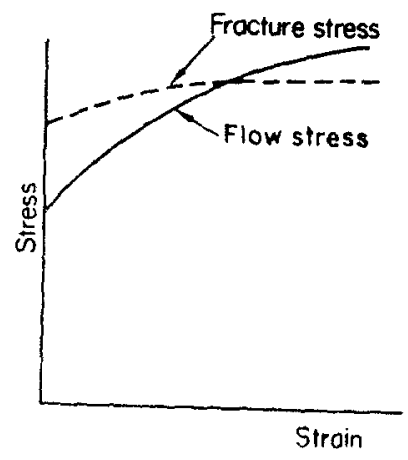

Figure 1. Illustration of the Ludwik-Davidenkov-Orowan hypothesis of ductile-brittle transition.

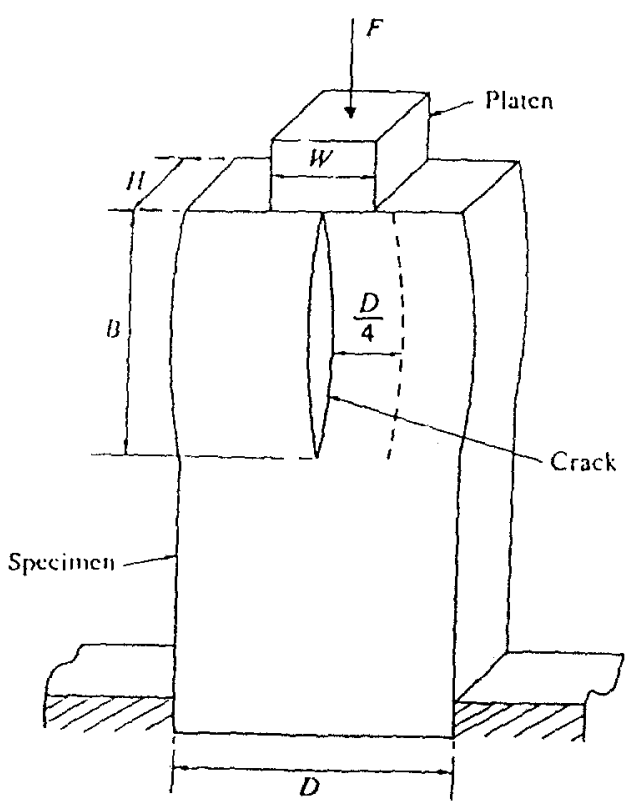

Figure 2. Geometry of the compression split specimen examined by Kendall. 


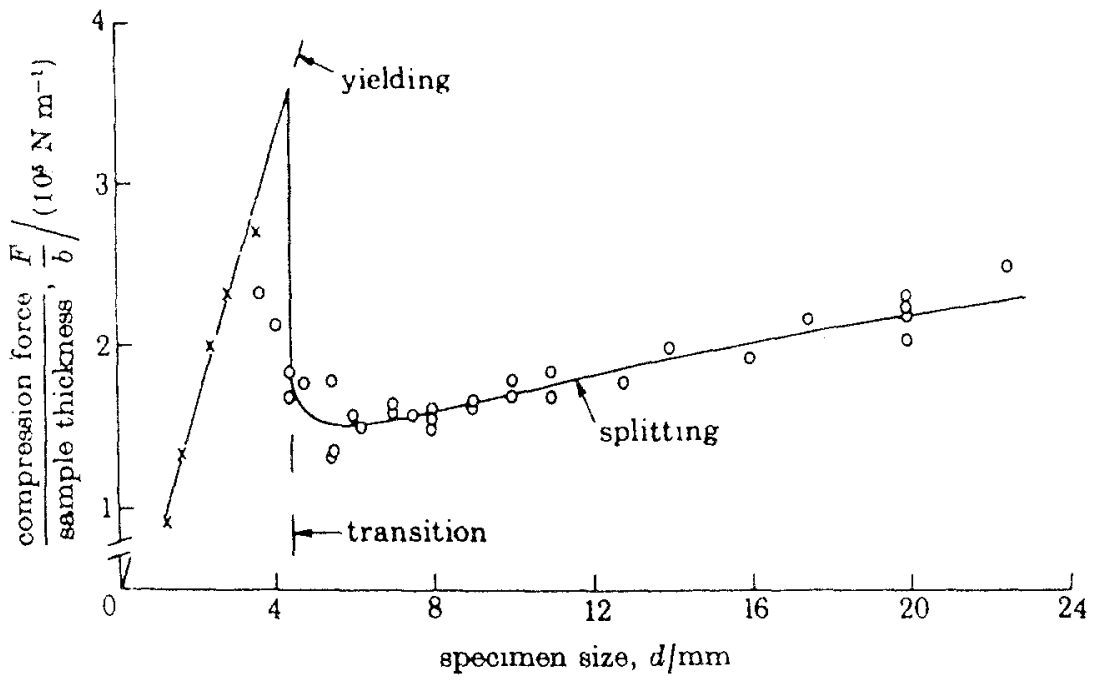

Figure 3. Compression results for different specimen sizes showing cracking ( $)$ of large samples and yielding $(x)$ of small samples of polystyrene.

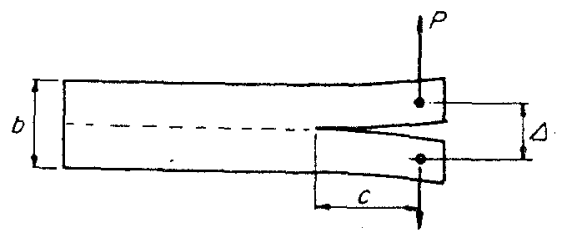

Figure 4. Double cantilever beam specimen. 